\title{
Elasticidade-renda das importações e Lei de Thirlwall: uma aplicação do método Markov-Switching para o Brasil (1995-2016)
}

\author{
Income elasticity of imports and Thirlwall's Law: \\ an application of the Markov-Switching method for Brazil (1995-2016)
}

\author{
Géssica Mathias Diniz \\ gessica92eco@gmail.com \\ Universidade Federal do Paraná (UFPR) \\ Diego Ferreira \\ diegoferreira.eco@gmail.com \\ Universidade Federal do Paraná (UFPR)
}

\begin{abstract}
Resumo: Este artigo propôs-se a analisar o comportamento da elasticidade renda das importações brasileiras, no período entre 1995 e 2016, e se existem evidências de nãolinearidade no que se refere à elasticidade. A elasticidade calculada permitiu testar a validade da Lei de Thirlwall, isto é, se o crescimento econômico do Brasil foi restrito pelo balanço de pagamentos. Para estimar a elasticidade foi o utilizado o método de Markov-Switching que permitiu a segmentação em regimes dos parâmetros estimados. Foram definidos dois regimes, para o primeiro a elasticidade foi de 4,78 com duração esperada de 1,3 trimestres, já para o segundo regime, obteve-se como resultado elasticidade igual a 0,62 e duração de 3,4 trimestres. Baseado nessas elasticidades confirmou-se a hipótese subjacente à Lei de Thirlwall, o balanço de pagamentos restringiu o crescimento econômico brasileiro no período.
\end{abstract}

Palavras-chaves: Elasticidade renda das importações; Markov-Switching; Balanço de pagamentos; Lei de Thirlwall; Brasil

\begin{abstract}
This article analyzes the behavior of income elasticity of Brazilian imports in the period 1995-2016 and whether there is evidence of non-linearity of the elasticity. The calculated elasticity allowed us to test the validity of the Thirlwall Law, if Brazil's economic growth has been constrained by the balance of payments. To estimate the elasticity this study employs Markov-Switching method that pemits the segmentation in regimes of the estimated parameters. Two regimes were defined. For the first, the elasticity was 4.78 with an expected duration of 1.3 quarters, while for the second regime, the elasticity was equal to 0.62 and duration of 3.4 quarters. Based on these elasticities, the hypothesis underlying the Thirlwall Law was confirmed, the balance of payments restricted the Brazilian economic growth in the period.
\end{abstract}

Key-words: Income elasticity of imports; Markov-Switching; Balance of payments; Thirlwall Law; Brazil 


\section{INTRODUÇÃO}

O Brasil destaca-se por sua representatividade no comércio internacional dentre os países latino-americanos, com o correspondente a um quinto do valor médio das exportações e das importações da região, no período entre 1995 e 2015 (ECLAC, 2016). Verifica-se que o crescimento econômico observado no país foi superior ao da América Latina até 2013, com taxas médias de crescimento do PIB de 3,20\% e 3,18\%, respectivamente (ECLAC, 2016). Passado esse período, o Brasil tem registrado taxas negativas e inferiores as apontadas para a região.

A fim de explorar o diferencial de taxas de crescimento entre países, Thirlwall (1979) apresentou uma proposta alternativa a abordagem dos modelos neoclássicos. Neste sentido, o autor propôs que essas diferenças se devem às restrições sobre a demanda, sendo o balanço de pagamentos o principal fator limitante. Com base em evidências empíricas, este identificou que a taxa de crescimento de vários países desenvolvidos se aproximava da taxa de crescimento das exportações dividida pela elasticidade-renda da demanda por importações, o que ele denomina como taxa de crescimento de equilíbrio do balanço de pagamentos.

Desde a disseminação dessa abordagem, diversos estudos têm sido realizados para identificar se o crescimento econômico de países ou macrorregiões foi de fato restrito pelo balanço de pagamentos. Para os casos latino-americano e brasileiro, pode-se citar Thirlwall \& Hussain (1982); López \& Cruz (2000); Bértola, Higachi \& Porcile (2002); Holland, Vieira \& Canuto (2004); Pacheco-López \& Thirlwall (2005); Cimoli, Porcile \& Rovira (2009); Alencar \& Strachman (2014).

Com base na teoria e na literatura existente, percebe-se o quão importante é analisar o crescimento pela ótica da demanda, em especial, de fatores concernentes ao balanço de pagamentos. Em vista disto e dada a expressividade econômica do Brasil no comércio exterior, este estudo analisa o comportamento da elasticidade-renda da demanda por importações brasileira entre 1995 e 2016. Para tanto, realiza-se uma estimação particionada em regimes endógenos através do Método de Markov-Switching e, a partir deles, são comparados o crescimento efetivo e o hipotético, com o intuito de verificar se o crescimento brasileiro foi efetivamente limitado pelo balanço de pagamentos nas últimas duas décadas.

Para atender tais objetivos, primeiramente são apresentados tanto o embasamento teórico acerca da Lei de Thirlwall quanto a literatura empírica sobre as aplicações desta para a América Latina e para o Brasil. No que tange ao método econométrico, uma equação 
multivariada de transição de regimes de Markov foi estimada a partir de dados sobre importação, Produto Interno Bruto (PIB) e câmbio brasileiros, obtidos no Banco Central do Brasil. O modelo de Markov Switching permite observar o comportamento variante no tempo das variáveis independentes revelando possíveis ciclos associados aos movimentos das economias doméstica e internacional.

O artigo está estruturado em três seções, além da presente introdução. A segunda se refere a revisão de literatura em que são apresentados a Lei de Thirlwall e alguns trabalhos que a testaram para a América Latina e o Brasil. Na terceira seção destacam-se os testes, o método e os principais resultados do trabalho. As considerações finais correspondem a seção quatro.

\section{REVISÃO DE LITERATURA}

Esta seção é dedicada à apresentação da ideia subjacente a Lei de Thirlwall e a literatura que testou a sua validade, especificamente, para a América Latina e para o Brasil.

\section{1 A Lei de Thirlwall}

Thirlwall (1979) apresentou uma proposta alternativa à dos neoclássicos quando se trata do diferencial na taxa de crescimento entre os países. Ao invés de explicá-la pela diferença no crescimento de fatores do lado da oferta e pela produtividade, o autor segue a abordagem keynesiana, com o enfoque nos diferenciais de progresso da demanda para explanar o crescimento econômico diverso dos países.

A renda é considerada a responsável por ajustes no balanço de pagamentos e não os preços como pressupõem os neoclássicos, pois, de acordo com Thirlwall (1979), isto não pode ser evidenciado empiricamente. $\mathrm{O}$ foco recai sobre as elasticidades renda das exportações e importações, requerendo mudanças na base produtiva doméstica que fomentem o setor exportador e promovam o crescimento econômico. O comércio exterior relacionado à demanda assume um papel de protagonismo nessa abordagem e isto a torna de grande valia para estudos em que o país analisado possui expressividade no setor.

Em um país que enfrente dificuldades no balanço de pagamentos, a demanda deve ser reduzida; a oferta nunca é totalmente utilizada; o investimento é desencorajado; o progresso tecnológico é lentamente reduzido; e os bens de um país em comparação com os bens 
estrangeiros tornam-se menos desejáveis, agravando ainda mais a balança de pagamentos (THIRLWALL, 1979).

Contudo, de acordo com Thirlwall (1979), ainda que se defenda o crescimento levado pelas exportações, taxas iguais de progresso das mesmas em diferentes países não resultarão em semelhantes taxas de crescimento do produto devido às distintas necessidades de importações destes.

A hipótese testada pelo autor é de que se o equilíbrio do balanço de pagamentos deve ser mantido, a taxa de crescimento de longo prazo de um país será determinada pela razão entre a taxa de crescimento das exportações e a elasticidade-renda da demanda por importações (THIRLWALL, 1979).

O equilíbrio do balanço de pagamentos é representado por:

$$
P_{d} X=P_{f} M E
$$

onde $X$ é a quantidade de exportações; $P_{d}$ é o preço das exportações em moeda doméstica; $M$ é a quantidade de importações; $P_{f}$ é o preço das importações em moeda estrangeira; $E$ é a taxa de câmbio (preço doméstico da moeda estrangeira).

Em uma economia que esteja crescendo, a condição de equilíbrio é que a taxa de crescimento do valor das exportações seja igual à taxa de crescimento do valor das importações:

$$
p_{d t}+x_{t}=p_{f t}+m_{t}+e_{t}
$$

A quantidade de importações demandada corresponde a uma função multiplicativa do preço das importações (em unidades de moeda doméstica), do preço dos substitutos às importações e da renda doméstica:

$$
M=b\left(P_{f} E / P_{d}\right)^{\Psi} Y^{\pi}
$$

onde $\Psi$ é a elasticidade preço da demanda por importações $(\Psi<0) ; Y$ é a renda doméstica e $\pi$ é a elasticidade renda das importações $(\pi>0)$. 
A quantidade de exportações demandada é expressa como uma função do preço das exportações (medida em moeda estrangeira), do preço dos bens que competem com as exportações e o nível de renda mundial.

$$
X=a\left(P_{d} / P_{f} E\right)^{\eta} Z_{t}^{\epsilon}
$$

onde $\eta$ é a elasticidade preço da demanda por exportações $(<0)$; $\varepsilon$ é a elasticidade renda das exportações $(>0)$.

Substituindo as equações de crescimento das exportações e importações (obtidas pela diferenciação com relação ao tempo das Equações 3 e 4 em logaritmo) na equação de equilíbrio do balanço de pagamento ao longo do tempo (Equação 2), chega-se a taxa de crescimento do produto condizente com esse equilíbrio.

$$
y_{B t}=\left[(1+\eta+\psi)\left(p_{d}+p_{f}+e\right)+\varepsilon(z)\right] / \pi
$$

Com a suposição adicional de que os preços relativos não mudam (ou a taxa de câmbio) no longo prazo, encontra-se a equação final da Lei de Thirlwall:

$$
y_{B t}=\varepsilon(z) / \pi=x / \pi
$$

onde, $y_{B t}$ é a taxa de crescimento da renda do país, $x$ é a taxa de crescimento das exportações e $\pi$ elasticidade-renda da demanda por importações. Quanto maior $\pi$, menor é a taxa de crescimento de equilíbrio do balanço de pagamentos (THIRLWALL, 2011).

Se a taxa de crescimento de equilíbrio do balanço de pagamentos pode ser expandida, pela maior atratividade das exportações e pela redução da elasticidade-renda da demanda por importações, a demanda pode crescer sem resultar em dificuldades ao balanço de pagamentos. No comparativo entre países emergentes e desenvolvidos, observa-se que os primeiros exportam matéria-prima e outras mercadorias básicas, para as quais os desenvolvidos têm baixa elasticidade-renda da demanda, enquanto os últimos exportam bens manufaturados que possuem alta elasticidade-renda da demanda por parte dos menos desenvolvidos (DAVIDSON, 1990). 
Segundo Thirlwall (1979), as diferenças nas taxas de crescimento de equilíbrio do balanço de pagamentos entre os países se devem às características dos bens produzidos que determinam a elasticidade-renda da demanda por exportações do país e a sua propensão a importar. Nesse sentido, países com baixa taxa de crescimento das exportações e alta elasticidade-renda da demanda por importações produzem bens relativamente não atrativos nos mercados doméstico e internacional.

A Lei de Thirlwall passou por modificações ao longo do tempo, com a inclusão de outras variáveis na análise, como o fluxo de capital, a relação dívida/PIB e o pagamento de juros sobre a dívida. Para mais detalhes ver Thirlwall \& Hussain (1982), McCombie \& Thirwall (1997), Moreno-Brid (1999), Barbosa-Filho (2001), Ferreira \& Canuto (2001), Moreno-Brid (2003)

\subsection{Aplicações da Lei de Thirlwall para a América Latina e o Brasil}

A adoção da Lei de Thirlwall tem resultado em estudos para vários países, em diferentes períodos e que se utilizam de diversos instrumentais metodológicos. Nesta subseção são apresentados trabalhos que tiveram como tema de pesquisa essa abordagem, com enfoque na literatura referente a América Latina e ao Brasil.

Thirlwall \& Hussain (1982) aplicaram o modelo básico (Equação 1) e o modelo estendido, que considera um desequilíbrio inicial da taxa de crescimento restrita do balanço de pagamentos e o crescimento do fluxo real de capital, para alguns países em desenvolvimento, inclusive o Brasil. No caso brasileiro, para dados de 1969 a 1978, verificouse que a taxa de crescimento efetiva foi maior que taxa predita pelo modelo básico $(0,095>0,040)$, o que indica um crescimento do fluxo real de capital superior ao do volume de exportações (THIRLWALL \& HUSSAIN, 1982).

Cimoli, Porcile \& Rovira (2009) elencaram como um dos objetivos de seu estudo a análise da evolução das exportações e da elasticidade-renda da demanda por exportações e importações na América Latina entre 1960 e 2004. Durante esse período, os autores observaram dois picos da elasticidade, nos anos de 1970 e de 1990 (superior a 2,5), mas com declínio na década de 1980 (aproximadamente 2,1). Esses pontos de máximo ocorreram quando a liberalização comercial e a sobrevalorização monetária predominavam como ações de política econômica em muitos países latino-americanos (CIMOLI, PORCILE \& ROVIRA, 2010). Os autores, no comparativo de alguns países latino-americanos com economias 
desenvolvidas e asiáticas, observaram que o parâmetro elasticidade-renda da demanda por importações foi superior para a América Latina, 2,13 contra uma média de 1,80 para as demais economias, ao longo desses 45 anos.

Em seu estudo sobre a evolução da elasticidade brasileira, Nakabashi (2007) identificou que ela variou entre 1947 e 2000 e que suas flutuações tendem a compensar a situação da economia externa. Desta forma, elasticidades não constantes são consideradas instrumento para suavização das restrições externas o que torna o processo de crescimento mais estável, pois em períodos favoráveis ela aumenta, caso contrário, tende a diminuir.

A análise da validade da Lei de Thirlwall no âmbito da América Latina, considerando todos os países da região ou somente uma amostra de países selecionados, foi o foco de muitos estudos, dentre eles Holland, Vieira \& Canuto (2004), Pacheco-López \& Thirlwall (2005), Esteves \& Correia (2012). Em geral, as evidências encontradas permitiram identificar que a situação latino-americana está de acordo com a Lei de Thirlwall.

No caso brasileiro, há também evidências que corroboram a proposição de uma relação de longo prazo entre o PIB, os termos de troca e a renda mundial, mesmo quando considerados diferentes períodos de tempo (LÓPEZ \& CRUZ (2000); BÉRTOLA, HIGACHI \& PORCILE (2002); BRITTO \& MCCOMBIE (2009); CIMOLI, PORCILE \& ROVIRA (2009); ALENCAR \& STRACHMAN (2014)).

No Quadro 1, é apresentada uma síntese dos objetivos, métodos e resultados obtidos nesses estudos citados acima.

Quadro 1 - Aplicações da Lei de Thirlwall e seus principais resultados.

\begin{tabular}{|c|c|c|c|c|}
\hline Trabalho & $\begin{array}{l}\text { Unidade de } \\
\text { análise }\end{array}$ & Objetivo & Método & Resultado \\
\hline $\begin{array}{l}\text { Países em } \\
\text { desenvolvimento }\end{array}$ & $\begin{array}{c}\text { Thirlwall \& } \\
\text { Hussain (1982) }\end{array}$ & $\begin{array}{c}\text { Aplicar o modelo } \\
\text { simples e o estendido } \\
\text { (com fluxo de capital) } \\
(1951-1978)\end{array}$ & $\begin{array}{l}\text { Literatura e } \\
\text { MQO }\end{array}$ & $\begin{array}{c}\text { Em equilíbrio, as } \\
\text { mudanças na } e \text { parecem } \\
\text { ter restringido os países } \\
\text { em seu crescimento em } \\
\text { cerca de } 0,6 \% \text { a.a., } \\
\text { enquanto as entradas de } \\
\text { capital permitiram que os } \\
\text { países crescessem um } \\
\text { pouco mais rapidamente } \\
\text { no comparativo com o } \\
\text { resultado do modelo } \\
\text { simples, em cerca de } \\
0,05 \% \text { a.a. }\end{array}$ \\
\hline América Latina & $\begin{array}{c}\text { López \& Cruz } \\
(2000)\end{array}$ & $\begin{array}{l}\text { Analisar se a taxa de } \\
\text { câmbio real é importante, } \\
\text { se sim, quão importante é } \\
\text { para a determinação da } \\
\text { taxa de crescimento com }\end{array}$ & $\begin{array}{l}\text { Vetores de } \\
\text { cointegração } \\
\text { e VAR }\end{array}$ & $\begin{array}{l}\text { A taxa de câmbio é } \\
\text { importante para o } \\
\text { equilíbrio externo, mas } \\
\text { uma taxa mais alta não } \\
\text { implica que o produto }\end{array}$ \\
\hline
\end{tabular}

Rev. Text. Econ., Florianópolis, v. 21 n. 1, p. 77 - 96, dez/mar. 2018 ISSN 2175-8085 


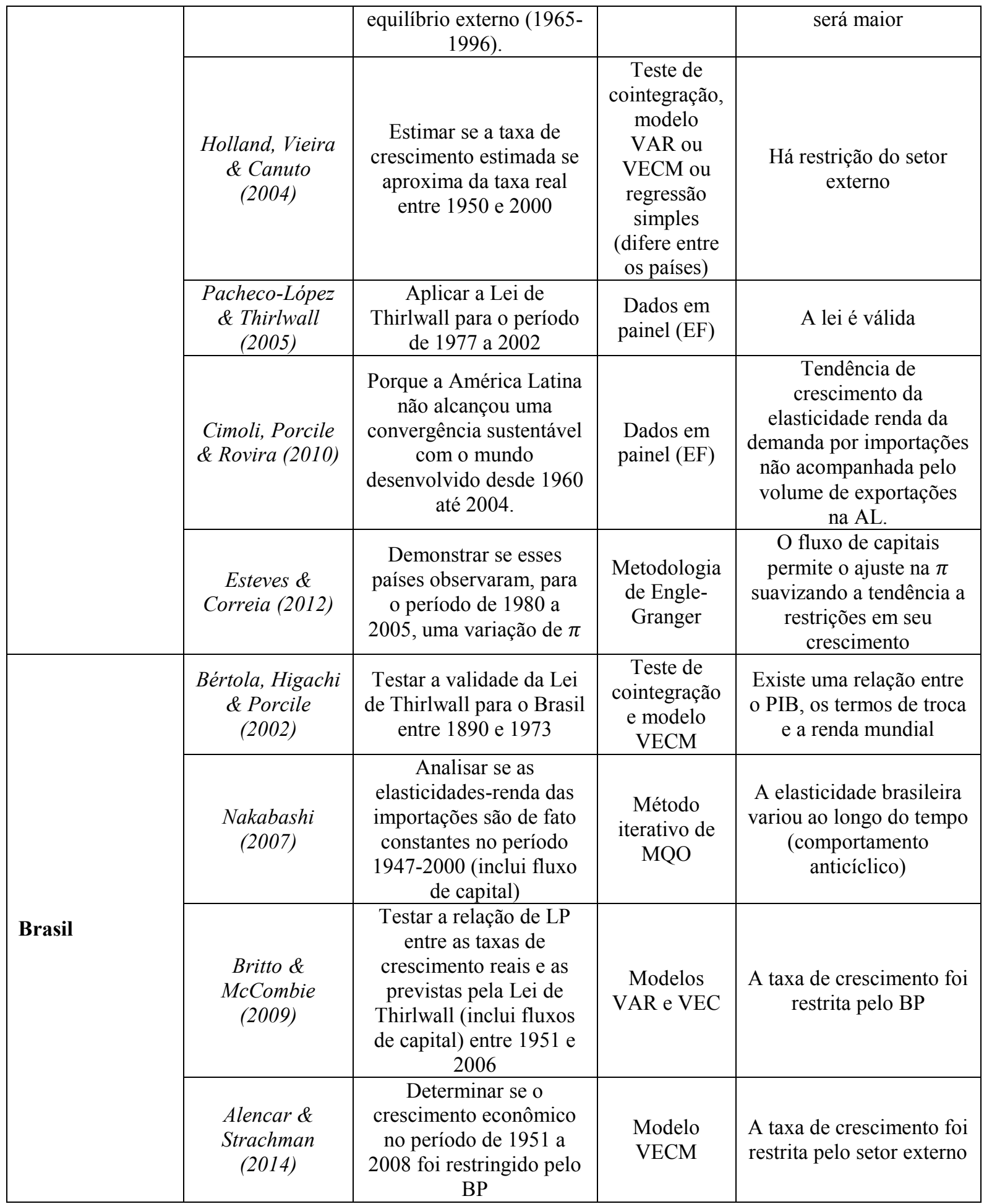

Fonte: Elaborado pelos autores.

\section{ELASTICIDADE-RENDA DA DEMANDA POR IMPORTAÇÕES DO BRASIL: UMA APLICAÇÃO DO MÉTODO MARKOV-SWITCHING}

Essa seção é dedicada a apresentação dos dados e dos resultados de testes realizados para a identificação da existência ou não de estacionariedade das séries. Além disso, Rev. Text. Econ., Florianópolis, v. 21 n. 1, p. 77 -96, dez/mar. 2018 ISSN 2175-8085 
apresentam-se o método de estimação do modelo de crescimento das importações e à descrição dos resultados obtidos com a regressão e pelo teste de validação da Lei de Thirlwall.

\subsection{Base de dados e testes}

Os dados de PIB, exportações, importações (em US\$ milhões) e o índice da taxa de câmbio real brasileiros foram extraídos da base de séries temporais do Banco Central do Brasil (2017). As séries mensais foram deflacionadas pelo Índice Nacional de Preços ao Consumidor Amplo (IPCA) (base: dezembro de 2010), obtido no Instituto Brasileiro de Geografia e Estatística (IBGE). Depois de deflacionados, os dados foram agrupados em trimestres. Os dados compreendem o período entre 1995:Q1 e 2016:Q4.

O modelo utilizado na literatura para mensurar a elasticidade segue abaixo:

$$
m_{t}=\psi(r e r)_{t}+\pi\left(y_{t}\right)+e_{t}
$$

onde $m$ é a taxa de crescimento do volume de importações, rer é a taxa de crescimento da taxa de câmbio real $\psi$ é a elasticidade preço da demanda por importações, $y$ é a taxa de crescimento da renda doméstica e $\pi$ é a elasticidade renda da demanda por importações.

Foram realizados dois testes de raiz unitária, Dickey Fuller Aumentado e PhillipPerron, os dois adotam como hipótese nula a não estacionariedade da série. Obteve-se como resultado que todas as séries possuem raiz unitária em nível (sem e com intercepto e com intercepto e tendência), enquanto as séries em primeira diferença são estacionárias (rejeitou-se a hipótese nula) (Tabela 1).

Tabela 1 - Resultados dos testes $A D F$ e Phillip-Perron para as variáveis em nível, em primeira diferença e para as taxas de crescimento.

\begin{tabular}{c|c|c|c}
\hline Variável & Restrição & $\boldsymbol{A D F}$ & $\boldsymbol{P P}$ \\
\hline \multirow{3}{*}{$\boldsymbol{M}$} & Sem intercepto & -0.5988 & 0.8243 \\
& Com intercepto & -1.4170 & -1.7224 \\
& Com intercepto e tendência & -1.2244 & -1.7346 \\
\hline \multirow{3}{*}{$\boldsymbol{X}$} & Sem intercepto & -0.3758 & -0.4760 \\
& Com intercepto & -1.8920 & -2.3417 \\
& Com intercepto e tendência & -1.9384 & -3.0290 \\
\hline \multirow{2}{*}{$\boldsymbol{P I B}$} & Sem intercepto & -1.2282 & -1.2067 \\
& Com intercepto & -1.4212 & -1.4212 \\
& Com intercepto e tendência & -1.4936 & -1.4936 \\
\hline
\end{tabular}




\begin{tabular}{|c|c|c|c|}
\hline$\Delta \boldsymbol{M}$ & $\begin{array}{c}\text { Sem intercepto } \\
\text { Com intercepto } \\
\text { Com intercepto e tendência }\end{array}$ & $\begin{array}{l}-4.3244 * * * \\
-4.3016 * * * \\
-4.3415 * * * \\
\end{array}$ & $\begin{array}{l}-8.3236 * * * \\
-8.2839 * * * \\
-8.2336 * * *\end{array}$ \\
\hline$\Delta X$ & $\begin{array}{c}\text { Sem intercepto } \\
\text { Com intercepto } \\
\text { Com intercepto e tendência }\end{array}$ & $\begin{array}{c}3.9775 * * * \\
-3.9517 * * * \\
-4.0265 * *\end{array}$ & $\begin{array}{l}-11.1892 * * * \\
-11.0807 * * * \\
-11.0689 * * *\end{array}$ \\
\hline$\triangle P I B$ & $\begin{array}{c}\text { Sem intercepto } \\
\text { Com intercepto } \\
\text { Com intercepto e tendência }\end{array}$ & $\begin{array}{l}-8.3372 * * * \\
-8.3428 * * * \\
-8.2975 * * *\end{array}$ & $\begin{array}{l}-8.3372 * * * \\
-8.3391 * * * \\
-8.2938 * * *\end{array}$ \\
\hline $\boldsymbol{G}_{M}$ & $\begin{array}{c}\text { Sem intercepto } \\
\text { Com intercepto } \\
\text { Com intercepto e tendência }\end{array}$ & $\begin{array}{l}-3.8050 * * * \\
-3.7888 * * * \\
-3.8191 * *\end{array}$ & $\begin{array}{l}-8.5697 * * * \\
-8.5370 * * * \\
-8.4860 * * *\end{array}$ \\
\hline $\boldsymbol{G}_{\boldsymbol{X}}$ & $\begin{array}{c}\text { Sem intercepto } \\
\text { Com intercepto } \\
\text { Com intercepto e tendência }\end{array}$ & $\begin{array}{c}-3.3055^{* * * *} \\
-3.2845 * * * \\
-3.3730 *\end{array}$ & $\begin{array}{l}-11.3067 * * * \\
-11.2009 * * * \\
-11.2692 * * *\end{array}$ \\
\hline $\boldsymbol{G}_{P I B}$ & $\begin{array}{c}\text { Sem intercepto } \\
\text { Com intercepto } \\
\text { Com intercepto e tendência }\end{array}$ & $\begin{array}{l}-8.5230 * * * \\
-8.5252 * * * \\
-8.4806 * * *\end{array}$ & $\begin{array}{l}-8.5089 * * * \\
-8.5104 * * * \\
-8.4648 * * *\end{array}$ \\
\hline
\end{tabular}

Nota: $M, X$ e PIB estão em nível. $\Delta$ representa a primeira diferença e $G_{M}, G_{X}$ e $G_{P I B}$ as taxas de crescimento das variáveis. *Significativo a $10 \%$. $* *$ Significativo a $5 \%$. $* * *$ Significativo a $1 \%$. Fonte: Elaborado pelos autores com base nos resultados obtidos no EViews 9.

Dado que no presente artigo são utilizadas taxas de crescimento para estimar o modelo de regressão e que tais taxas são resultantes da diferença entre o logaritmo natural das variáveis em $t$ e $t-1$, tem-se que as séries de taxa de crescimento não possuem raiz unitária, conforme resultados dos testes ADF e PP.

\subsection{0 método}

O modelo de regressão Markov-Switching é utilizado quando os parâmetros do modelo variam em diferentes regimes não-observados, é um modelo em que a não-linearidade surge a partir de mudanças discretas entre regimes (EVIEWS 8.1, 2014). Devido a uma mudança na política econômica, um período de recessão ou boom, etc. o comportamento do parâmetro pode diferir em cada estágio (SÁNCHEZ, 2015).

No modelo, a variável de interesse $y_{t}$ segue um processo que depende do valor de uma variável discreta não observada $s_{t}$. Assume-se que existam $M$ possíveis regimes e que se está no regime $m$ no período $t$ quando $s_{t}=m$, para $m=1, \ldots, M$.

O modelo Switching considera que exista um modelo de regressão associado a cada regime, isto é, existem diferentes valores para os parâmetros das variáveis que forem consideradas não lineares no modelo, os quais serão proporcionais ao número de regimes. A escolha de tal método para este estudo se deve justamente à possibilidade de identificação de comportamento não linear das variáveis independentes, permitindo que se faça um paralelo Rev. Text. Econ., Florianópolis, v. 21 n. 1, p. 77 -96, dez/mar. 2018 ISSN 2175-8085 
entre essas mudanças e as flutuações econômicas e políticas tanto nacionais quanto internacionais. Morais \& Portugal (2005) justificam em seu estudo sobre a demanda por importações brasileira o uso do modelo de Markov-Switching pela relação existente entre regimes de política de importação no Brasil e oscilações cíclicas de liquidez internacional, o que condiciona diferentes formas de controle das importações. Tal modelo facilita o acompanhamento dessa relação pela identificação de regimes.

Dados os regressores $X_{t}$ e $Z_{t}$, a média condicional de $y_{t}$ no regime $m$ possui a seguinte especificação linear:

$$
\mu_{t}(m)=X_{t}^{\prime \beta_{m}}+Z_{t}^{\prime} \gamma
$$

onde $\beta_{m}$ e $\gamma$ são $K_{X}$ e $K_{Z}$ vetores de coeficientes. Os coeficientes do primeiro termo são indexados pelo regime enquanto os coeficientes do segundo termo não variam entre os regimes.

Assume-se que os erros da regressão são normalmente distribuídos com variância dependente do regime, pois, quando $s_{t}=m$, o desvio padrão $(\sigma)$ é dependente do regime, $\operatorname{logo} \sigma(m)=\sigma_{t}$. Assim, o modelo de regressão é especificado como:

$$
y_{t}=\mu_{t}(m)+\sigma(m) \epsilon_{t}
$$

A contribuição da verossimilhança para uma dada observação deve ser formada pela ponderação da função densidade em cada um dos regimes pela probabilidade de que a um passo atrás se estivesse nesse regime, ou seja:

$$
L_{t}(\beta, \gamma, \sigma, \delta)=\sum_{m=1}^{M} \frac{1}{\sigma_{m}} \phi\left(\frac{y_{t}-\mu_{t}(m)}{\sigma(m)}\right) * P\left(s_{t}=m \mid \zeta_{t-1}, \delta\right)
$$

onde $\beta=\left(\beta_{1}, \ldots, \beta_{M}\right), \sigma=\left(\sigma_{1}, \ldots, \sigma_{M}\right), \delta$ são os parâmetros que determinam o regime de probabilidades, $\phi$ (.) é a função densidade normal, e $\zeta_{t-1}$ é o conjunto de informações em $t-1$.

A função log-verossimilhança é especificada como um processo normal mixture, de modo que: 


$$
l(\beta, \gamma, \sigma, \delta)=\sum_{t=1}^{T} \log \left\{\sum_{m=1}^{M} \frac{1}{\sigma_{m}} \phi\left(\frac{y_{t}-\mu_{t}(m)}{\sigma(m)}\right) * P\left(s_{t}=m \mid \zeta_{t-1}, \delta\right)\right\}
$$

As probabilidades são obtidas por um logit multinomial, sendo que a especificação para cada linha $i$ da matriz é dada por:

$$
p_{i j}\left(G_{t-1}, \delta_{i}\right)=\frac{\exp \left(G_{t-1}^{\prime} \delta_{i j}\right)}{\sum_{s=1}^{M} \exp \left(G_{t-1}^{\prime} \delta_{i s}\right)}
$$

para $j=1, \ldots, M$ e $i=1, \ldots, M$ com normalização $\delta_{i, M}=0$. As probabilidades podem ou não ser variantes no tempo, mas, geralmente, os modelos Markov Switching são especificados com probabilidades de transição constantes dentro de cada regime, logo, $G_{t-1}$ contém somente uma constante.

O modelo de regressão Markov Switching estende a estrutura de probabilidade exógena simples ao incluir o processo de Markov de primeira ordem para o regime de probabilidades. A suposição do processo markoviano requer que a probabilidade de se estar em um regime dependa do estágio anterior, isto é

$$
P\left(s_{t}=j \mid s_{t-1}=i\right)=p_{i j}(t)
$$

O tempo de transição entre regimes e a duração de cada um são ambos aleatórios. A probabilidade de transição $\left(p_{i j}\right)$ é a probabilidade de estar no estado $j$ no período atual dado que o processo estava em um regime $i$ no período anterior, como se observa na matriz de transição abaixo, que se refere a um modelo com três regimes

$$
p(t)=\left[\begin{array}{ccc}
p_{11}(t) & \ldots & p_{1 M}(t) \\
\cdot & \ldots & \cdot \\
p_{M 1}(t) & \ldots & p_{M M}(t)
\end{array}\right]
$$

Existem três formas de estimação do regime de probabilidades: um passo atrás, filtrado e suavizado. No presente trabalho, a estimação suavizada foi aplicada visto que esta incorpora todas as informações no período final, $\zeta_{T}$, diferentemente da estimativa filtrada, que reúne somente informações contemporâneas, $\zeta_{t}$.

\subsection{Resultados e discussões}

Rev. Text. Econ., Florianópolis, v. 21 n. 1, p. 77 -96, dez/mar. 2018 ISSN 2175-8085 
O Brasil é um dos diversos países que formam a América Latina e se destaca pela expressiva participação no comércio e no produto da região, como se pode observar na Figura 1. As exportações brasileiras corresponderam, em média, no período entre 1995 e 2015, a $20 \%$ das exportações latino-americanas e a mais de $40 \%$ do PIB da região. O país possui uma pauta exportadora em que predominam bens básicos e manufaturados, em 2016, os produtos primários representaram 46\% do total exportado (MDIC, 2017).

Figura 1 - Participação das exportações e do PIB brasileiros no total da América Latina $(1995-2015)(\mathrm{em} \%)$.

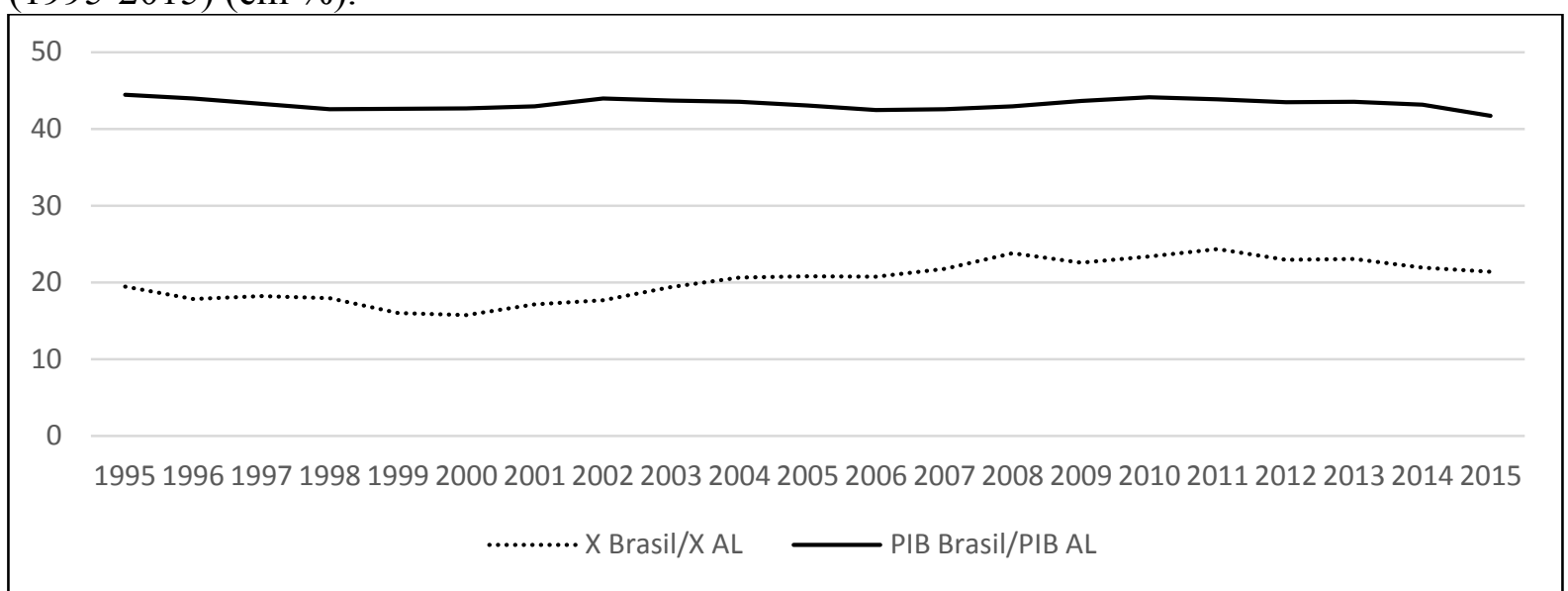

Fonte: Elaborada pelos autores com base nos dados da ECLAC (2016).

Após a implementação do programa de estabilização econômica proposto pelo Plano Real, em 1994, e da liberalização comercial, o Brasil passou por um período de grande influxo de importações, superando as exportações, devido à manutenção da paridade real dólar (sobrevalorização do real). Em 1999, com a adoção do câmbio flutuante, o real foi desvalorizado e o volume monetário das exportações passou a crescer acima das importações. As exportações cresceram 36.3\% entre o primeiro trimestre de 1995 e o quarto trimestres de 2016 (BCB, 2017).

O produto brasileiro tem acompanhado as flutuações das exportações, em que os vales das séries refletem as diversas crises que ocorreram ao longo desse período: mexicana (19941995), asiática (1997-1998), russa (1998), brasileira (1999), argentina (2001-2002) e norteamericana (2001 e 2009). Em 2015, o PIB passou por nova redução, a qual se deve a crise política e econômica instaurada no país.

A relevância das exportações no produto brasileiro pode ser observada na Figura 2, com essa proporção superando a participação das importações. O somatório dessas variáveis 
já correspondeu, em média, a 21\% do produto brasileiro, entre 2001 e 2008, período marcado pela entrada da China na Organização Mundial do Comércio (OMC), já de 2009 até o período mais recente essa proporção caiu para 18\% (PEREIRA, 2016). De acordo com a autora, entre 2008 e 2014, o volume de comércio de bens e serviços e o PIB mundial tiveram seu crescimento desacelerado, como resultado do lento progresso das principais economias do mundo. O Brasil é suscetível a essas mudanças o que contribui para explicar a redução de seu comércio.

Figura 2 - Participação das exportações e das importações no PIB brasileiro (1995-2016) (em

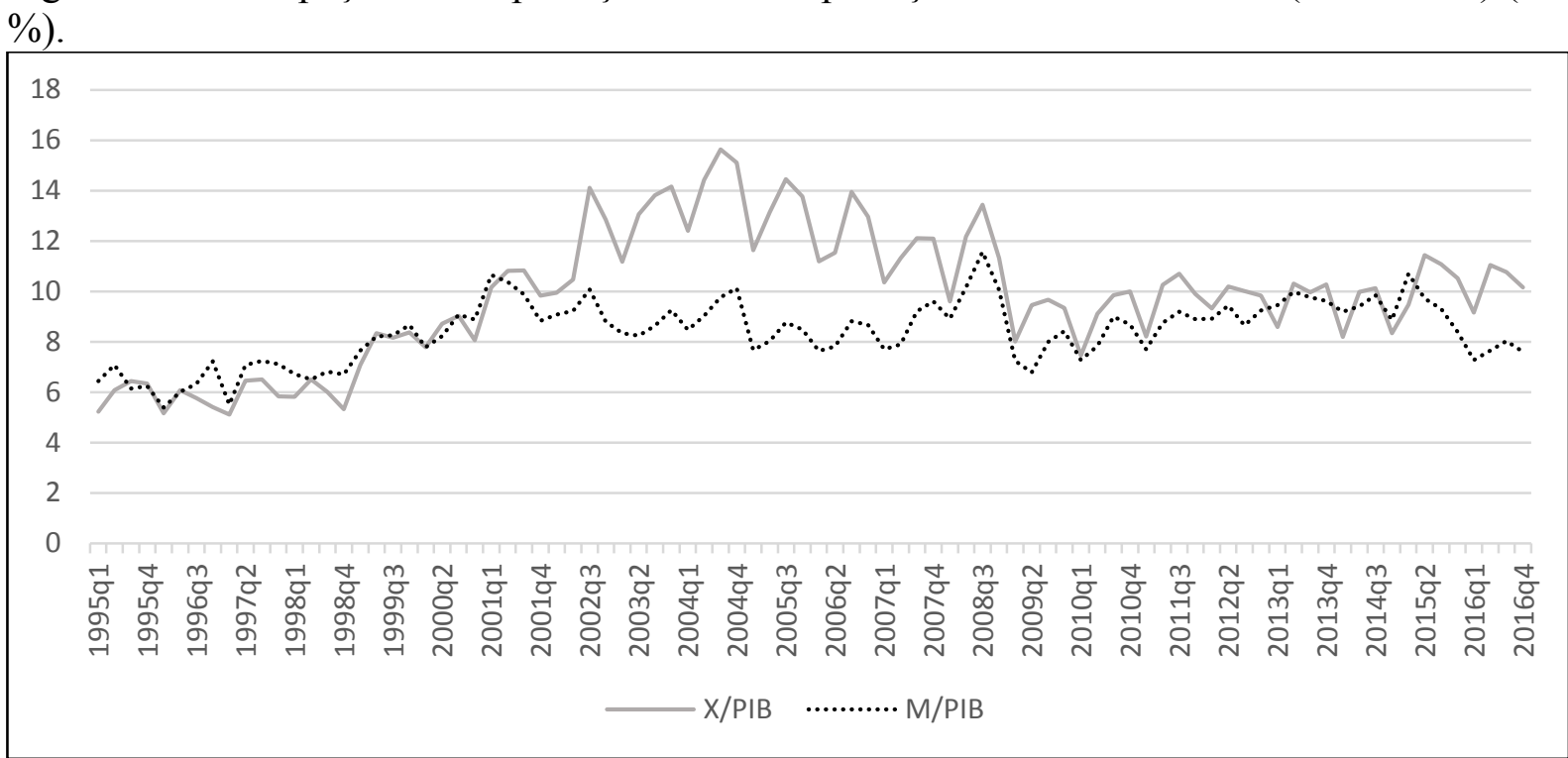

Fonte: Elaborada pelos autores com base nos dados do BCB (2017).

O saldo da balança comercial voltou a tornar-se deficitário, em 2014, mas se recuperou em 2015, com à queda das importações a um nível inferior ao das exportações. A diminuição das exportações brasileiras, observada desde 2012, pode ser explicada, em particular, pela desaceleração da economia chinesa iniciada em 2011 (redução da taxa de crescimento do PIB, de 10,64\%, em 2010, para 7,86\%, em 2012) contribuindo para a redução do preço das commodities. A China é a principal parceira comercial do Brasil como importadora de bens primários, principalmente, soja, minério de ferro e petróleo e a segunda maior exportadora de bens para o Brasil, principalmente, manufaturados, conforme dados de 2016 (MDIC, 2017). Desde 2009, o país assumiu o lugar dos Estados Unidos como maior importador dos produtos brasileiros (CONTI \& BILKSTAD, 2017). A China consumiu 19\% das exportações brasileiras e forneceu 17\% das importações brasileiras, em 2016, mas essas parcelas já chegaram a 22\% e 18\%, em 2013 (MDIC, 2017). 
Ao estimar a elasticidade renda das importações (Equação 7) via método de MarkovSwitching foi possível definir apenas dois regimes para estimar o modelo de regressão, considerando a não-linearidade do coeficiente associado à variável taxa de crescimento do produto (G_PIB), enquanto $\psi$ (elasticidade preço da demanda por importações) permaneceu constante nos dois regimes, pois não houve evidência de não-linearidade deste parâmetro.

Observa-se nas Equações 15 e 16 que no primeiro regime a elasticidade renda das importações foi superior a identificada no segundo regime, mas em ambos os casos esses coeficientes foram significativos a $1 \%$. No mesmo sentido seguem os coeficientes dos resíduos. Já o parâmetro elasticidade preço da demanda por importações não pôde ser considerado diferente de zero, ao nível de significância de $10 \%$.

$$
\begin{aligned}
& m_{t}=0.0252\left(\operatorname{rer}_{t}\right)+4.7764\left(y_{t}\right)+0.8379 e_{t} \\
& (0.0799) \quad(0.1751)^{* * *} \quad(0.2931)^{* * *} \\
& m_{t}=0.0252\left(\text { rer }_{t}\right)+0.6194\left(y_{t}\right)+2.1395 e_{t} \\
& (0.0799) \quad(0.1182)^{* * *} \quad(0.0981)^{* * *}
\end{aligned}
$$

Os resultados da matriz de transição revelaram que a probabilidade de se estar no regime 2 dado que se estava no regime 1 ou até mesmo de permanecer no segundo estágio é superior à probabilidade de mudar ou continuar no regime 1 , acima de $70 \%$ e menos de $30 \%$, respectivamente. Esses valores são refletidos na duração esperada de cada regime, sendo maior para o segundo regime, em média, 3,4 trimestres, enquanto o primeiro deve perdurar por 1,3 trimestres (Figura 3).

Figura 3 - Probabilidades de transição suavizadas.

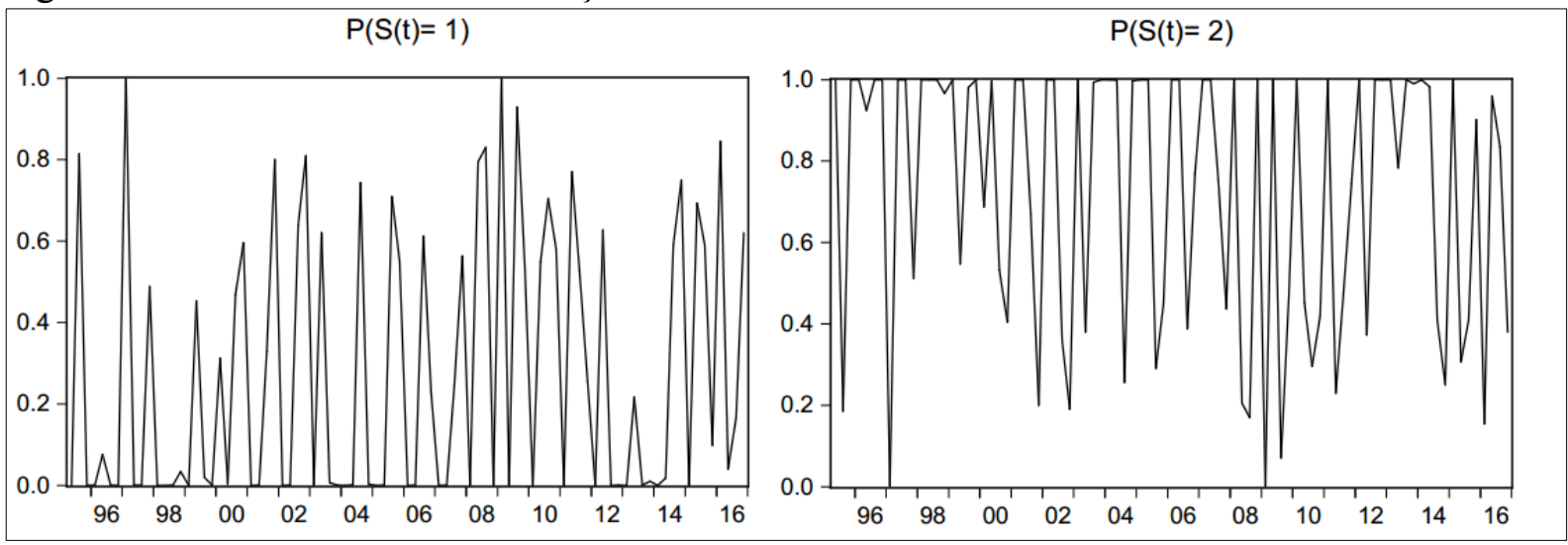

Fonte: Elaborada pelos autores com base no Output obtido no EViews 9. 
Como são considerados dados trimestrais há certa dificuldade de se observar a transição entre os regimes, mas percebe-se que ao longo desses 21 anos as elasticidades acompanharam as flutuações da economia doméstica e internacional. Alguns dos fatores que poderiam explicar a evolução no tempo das elasticidades renda das importações real e calculada se devem, entre outros aspectos a variação da taxa de câmbio real e seus efeitos sobre a balança comercial e o equilíbrio no BP e a entrada e a saída de capitais pela conta capital e pelo balanço de serviços (NAKABASHI, 2003). Em períodos de maior influxo de capital e valorização da moeda doméstica a elasticidade tende a crescer, caso contrário, há retração desse parâmetro.

Com base nas elasticidades renda das importações obtidas pelo método MarkovSwitching, pôde-se testar a validade da Lei de Thirlwall para o Brasil, no período de 1995 a 2016. Os valores do parâmetro foram substituídos na Equação 6 do modelo ${ }^{1}$ e assim chegouse ao produto hipotético, na sequência realizou-se uma estimação por MQO, em que a variável dependente é a taxa de crescimento do produto estimada:

$$
y_{t}^{e s t}=\alpha y_{t}^{o b s}+e_{t}
$$

Se o coeficiente $\alpha$ for estatisticamente significativo pode-se rejeitar a hipótese nula de que o mesmo é igual a zero e por meio da estatística $t$ testa-se a hipótese de que ele seja igual a 1. Na Tabela 1 são apresentados os resultados obtidos com a regressão e as estatísticas para verificar se a taxa de crescimento efetiva é estatisticamente igual à taxa calculada.

Tabela 1 - Regressão MQO e estatísticas

\begin{tabular}{c|c}
\hline$\alpha$ & $0.7892^{* * *}$ \\
Desvio padrão & $(0.1918)$ \\
\hline Estatística $t$, para $\mathrm{H}_{0}=1$ & -1.0999 \\
\hline
\end{tabular}

Nota: ***Significativo a 1\%. Fonte: Elaborada pelos autores com base nos resultados do Eviews 9.

Pôde-se rejeitar que o parâmetro seja zero (com nível de significância de 1\%) e o teste $t$ de Student não permitiu a rejeição da hipótese nula de que o coeficiente de $y_{t}^{o b s}$ é igual a 1 , com nível de significância de 10\%. Logo, observa-se que o balanço de pagamentos restringiu o crescimento econômico brasileiro entre 1995 e 2016.

\footnotetext{
${ }^{1}$ A elasticidade renda de cada trimestre foi obtida pela média ponderada das elasticidades de cada regime por suas probabilidades de ocorrência.

Rev. Text. Econ., Florianópolis, v. 21 n. 1, p. 77 -96, dez/mar. 2018 ISSN 2175-8085
} 
Os resultados deste estudo demostraram que as flutuações do comércio brasileiro têm efeitos sobre o crescimento econômico do país e tais oscilações dependem do comportamento da economia mundial e das políticas econômicas definidas no âmbito doméstico. Logo, o que pode ser feito para fomentar o crescimento do país, que está ao alcance dos formuladores de política econômica é utilizar instrumentos, como câmbio, juros, tarifas, entre outros, para estimular as exportações e retrair a demanda por importações.

\section{CONSIDERAÇÕES FINAIS}

Este artigo teve por objetivo identificar o comportamento da elasticidade renda das importações brasileiras (1995-2016) com o uso do método de regressão Markov-Switching e testar a validade da Lei de Thirlwall com base nas elasticidades calculadas.

O resultado obtido para a elasticidade renda das importações revelou a existência de dois regimes distintos, que refletem as flutuações das economias doméstica e internacional no período. A possibilidade de análise do comportamento não linear do parâmetro, via estimação de um modelo Markov-Switching, permite testar a validade da Lei de Thirlwall na presença de diferentes regimes para a elasticidade-preço das importações e denota, assim, a relevância deste trabalho. Até então, poucos estudos têm dedicado atenção a flutuações da elasticidade ao longo do tempo com tal objetivo (Nakabashi (2007) - Brasil; Pacheco-López \& Thirlwall (2005) - América Latina e; Cimoli, Porcile \& Rovira (2010) - América Latina, Ásia e países desenvolvidos).

Mas ainda que para o caso brasileiro Nakabashi (2007) tenha se preocupado com as variações da elasticidade renda das importações entre 1947 e 2000, a sua avaliação foi pautada em regressões de MQO particionadas em subperíodos de quatro anos com dummies associadas a cada um deles. $\mathrm{O}$ uso desse método reduz os graus de liberdade, nesse sentido, o método de Markov-Switching mostra-se mais eficiente ao permitir a segmentação dos parâmetros em regimes com uma única regressão sem perder tantos graus de liberdade.

O comércio desempenha um importante papel para o crescimento econômico de um país, dado que um saldo comercial superavitário permite a expansão do produto agregado. Ao testar a veracidade do argumento de Thirlwall (1979), percebeu-se que ele é válido no caso do Brasil, alinhando-se às conclusões obtidas em outros trabalhos (López \& Cruz (2000); Bértola, Higachi \& Porcile (2002); Britto \& McCombie (2009, 2013); Cimoli, Porcile \& Rovira (2009); Alencar \& Strachman (2014)). 
A validação da lei para o Brasil, bem como a identificação da existência de regimes de elasticidade renda das importações, pode servir como referência para a formulação de políticas por parte do governo com a finalidade de estimular o setor exportador e reduzir a dependência doméstica por importações, o que colaboraria para o fomento do crescimento econômico do país ao estimular o superávit da balança comercial.

Para um estudo futuro, poderia estender-se o modelo básico de Thirwall (1979), incorporando no modelo de regressão das importações a formação bruta de capital fixo e o consumo das famílias, como feito por Gouvêa \& Schettini (2011), a fim de avaliar as implicações da inclusão dessas variáveis sobre os movimentos da elasticidade no tempo.

\section{REFERÊNCIAS BIBLIOGRÁFICAS}

ALENCAR, D. A.; STRACHMAN, E. Balance-of-payments-constrained growth in Brazil: 1951-2008. Journal of Post Keynesian Economics, 36:4, p. 673-698, 2014.

BANCO CENTRAL DO BRASIL (BCB, 2017). Sistema Gerenciador de Séries Temporais (v2.1). Disponível

em: $<$ https://www3.bcb.gov.br/sgspub/localizarseries/localizarSeries.do?method=prepararTelaLoc alizarSeries>. Acesso em: 20 mai. 2017.

BARBOSA-FILHO, N. The balance of payments constraint: from balanced trade to sustainable debt. BNL Quarterly Review, Dec/2001.

BÉRTOLA, L., HIGACHI, H.; PORCILE, G. Balance-of-payments-constrained growth in Brazil: a test of Thirlwall's Law, 1890-1973. Journal of Post-Keynesian Economics, v. 25, n. 1, p. 123-140, 2002.

BRITTO, G.; MCCOMBIE, J. S. L. Thirlwall's law and the long term equilibrium growth rate: an application to Brazil. Journal of Post Keynesian Economics, v. 32, n. 1, p. 115-136, 2009.

BRITTO, G.; MCCOMBIE, J. S. L. Thirlwall's Law and the Long-Term Equilibrium Growth Rate: An Application to Brazil. An addendum. Journal of Post Keynesian Economics, v. 35, n. 4, 2013.

CIMOLI, M.; PORCILE, G.; ROVIRA, S. Structural Change and the BOP-constraint: Why did Latin America Fail to Converge? Cambridge Journal of Economics, v. 34, n. 2, p. 389411, 2009.

CONTI, B. de; BILKSTAD, N. Impactos da economia chinesa sobre a brasileira no início do século XXI. Texto para Discussão: Unicamp. IE, Campinas, n. 292, abr./2017.

DAVIDSON, P. A Lei de Thirlwall. Revista de Economia Política, v. 10, n. 4 (40), out.dez./1990. 
ECONOMIC COMMISSION FOR LATIN AMERICA AND THE CARIBBEAN (ECLAC). CEPALSTAT Database and Statistical Publications, 2016. Disponível em: $<$ http://estadisticas.cepal.org/cepalstat/WEB_CEPALSTAT/estadisticasIndicadores.asp?idiom $\mathrm{a}=\mathrm{i}>$. Acesso em: 20 mai. 2017.

ESTEVES, L. E.; CORREIA, F. M. Crescimento econômico e Lei de Thirlwall: uma análise para economias latino-americanas. Análise Econômica, v. 30, n. 57, p. 131-150, 2012.

EVIEWS. EViews 8.1 User's Guide II. IHC Global Inc., 2014.

FERREIRA, A.; CANUTO, O. Thirlwall's Law and foreign capital in Brazil. Momento Econômico, jan./2003.

GOUVÊA, R. R.; SCHETTINI, B. P. Estimativas econométricas para as importações agregadas com dados das contas nacionais trimestrais - 1996-2010. Texto para Discussão: IPEA, n. 1683, 2011.

HOLLAND, M., VIEIRA, F.; CANUTO, O. Economic growth and the balance-of-payments constraint in Latin America, Investigación Económica, v. LXIII, p. 45-74, 2004.

INSTITUTO BRASILEIRO DE GEOGRAFIA E ESTATÍSTICA (IBGE, 2017). Sistema Nacional de Índices de Preços ao Consumidor. Disponível em: < http://www.ibge.gov.br/home/estatistica/indicadores/precos/inpc_ipca/defaultseriesHist.shtm> . Acesso em: 20 mai. 2017.

LÓPEZ, J. G.; CRUZ, A. B. Thirlwall's law and Beyond: the Latin American Experience. Journal of Post Keynesian Economics, v. 22, n. 3, p. 477-495, 2000.

MCCOMBIE, J. S. L. 'Thirlwall's Law' and balance of payments constrained growth - a comment on the debate. Applied Economics, v. 21, n. 5, p. 611-629, 1989.

MCCOMBIE J.S.L.; THIRLWALL A.P. Economic growth and the balance of payments constraint revisited. In: ARESTIS P., PALMA P. AND SAWYER M. (eds.). Markets, unemployment and economic policy: essays in honour of G. Harcourt, v. 2. London: Edward Elgar, 1997.

MINISTÉRIO DA INDÚSTRIA, COMÉRCIO EXTERIOR E SERVIÇOS (MDIC). Balança comercial brasileira: acumulado do ano. 2017. Disponível em: $<$ http://www.mdic.gov.br/index.php/comercio-exterior/estatisticas-de-comercioexterior/balanca-comercial-brasileira-acumulado-do-ano>. Acesso em: 21 jul. 2017.

MORAIS, I. A. C. de; PORTUGAL, M. S. A Markov Switching model for the Brazilian demand for imports: analyzing the import substitution process in Brazil. Brazilian Review of Econometrics, v. 25, n. 2, p. 173-218, 2005.

MORENO-BRID, J. C. On Capital Inflows and the balance-of-payments constrained growth model. Journal of Post Keynesian Economics, v. 21, n. 2, p. 283-298, 1999. 
Capital flows, interest payments and the balance of payments constrained growth $\overline{\text { model: }}$ a theoretical and empirical analysis. Metroeconomica, May/2003.

NAKABASHI, L. Crescimento com restrição no Brasil: uma abordagem com diferentes elasticidades. Texto para Discussão. Belo Horizonte: Cedeplar/UFMG, n. 203, p. 1-18, 2003.

. O Modelo de Thirlwall com variações nas elasticidades. Economia e Sociedade, Campinas, v. 16, n. 1 (29), p. 93-110, abr./ 2007

PACHECO-LÓPEZ, P.; THIRLWALL, A.P. Trade Liberalisation, the Income Elasticity of Demand for Imports and Growth in Latin America. Department of Economics Discussion Paper, University of Kent, n. 05,06, 2005.

PEREIRA, L. B. V. O lento crescimento do comércio mundial. Conjuntura Econômica: Comércio Exterior. FGV IBRE, jul./2016.

SÁNCHEZ, G. Introduction to Markov-switching regression models using the mswitch command. StataCorp, Espanha, oct./ 2015.

THIRLWALL, A. P. The balance of payments constraint as an explanation of international growth rate differences. Banca Nazionale del Lavoro Quaterly Review, n. 128, 1979.

- Balance of payments constrained growth models: history and overview. PSL Quarterly Review, v. 64, n. 259, p. 307-351, 2011.

THIRLWALL, A. P.; HUSSAIN, M. N. The balance of payments constraint, capital flows and growth rate differences between developing countries. Oxford Economic Papers, New Series, v. 34, n. 3, p. 498-510, nov./ 1982. 